\title{
Interactive mobile robotic drinking glasses
}

François Rey, Michele Leidi, Francesco Mondada

\begin{abstract}
The central idea behind this attempt is to merge common objects and robotics to obtain a new type of interactive artefact, we call robjects. Robjects provide services to everyday life and can be controlled by the user in a very intuitive way. Robjects take rarely the initiative, have few decisional autonomy. Most of their activity is centered and controlled by the interaction with the user.

To test this concept in a concrete experiment we decided to include mobile robotic technology into drinking glasses to improve their service on a table. Making them mobile and interactive brings a new dimension to the table, simplifies service and improves security.

The system works in the following manner : when a user starts filling a glass, the other empty glasses come closer to get filled too and then go back to their initial position. Owners of the glasses can refuse to fill their glasses by simply bringing back the glass on its initial position as soon as the glass starts to move.

The system has been prototyped and tested. The analysis of the interaction of some users shows strengths in interactivity and weaknesses in the speed of the system and in reliability of the actual prototype.
\end{abstract}

\section{Introduction}

\subsection{Goal of the project}

The goal of this project is to develop a swarm of "robot objects", we call robjects. Robjects are fully autonomous robots with the shape of common objects. They interact with users in a very intuitive way to provide services in everyday life situations. Interaction is fully based on a multimodal natural interface applied to the object it-

Laboratoire de Systèmes Robotiques - École Polytechnique Fédérale de Lausanne firstname. lastnamedepfl.ch 

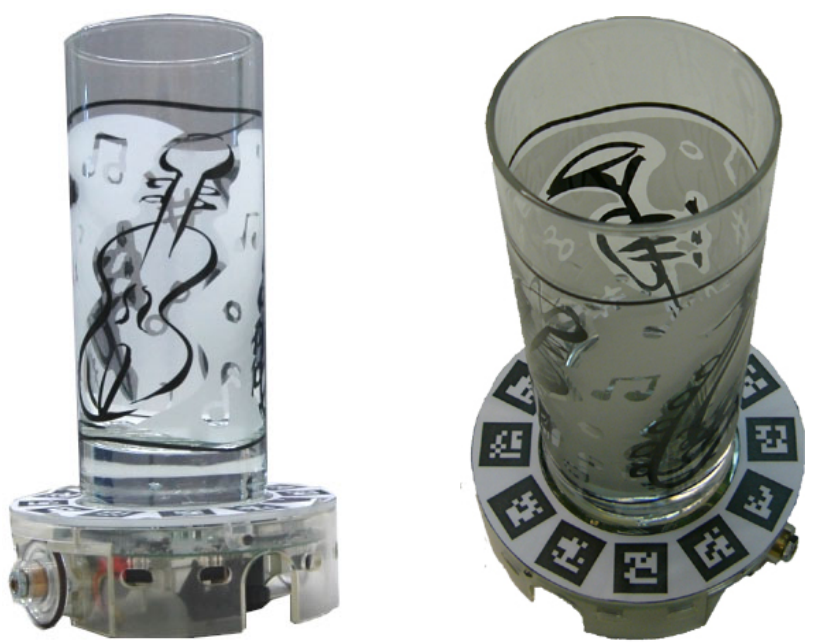

Fig. 1 Interactive and mobile drinking glasses merging the role of glasses and mobile robotic technology. Lateral and top views.

self. Touching, pushing, grasping, making gestures or a sequence of actions are the basic elements of a simple interaction like the one we present here. The goal of the interaction is to control the robot and bring him to do what the user desires. This concept is in opposition to most domestic autonomous robot concepts (often with an humanoid form) proposed by the scientific community and fits better with people expectation, at least in Europe [1].

The scenario presented in this paper is the lunch of a family with two adults and two children. Everybody drink water and parents drink wine too. Water and wine are in glasses of different shapes. All glasses together form a swarm where each glass can move and interact with users and other glasses. When a glass is filled, it communicate this event to the swarm of glasses. The location of each glass is given by a vision system placed above the table. Glasses that have the same content and are nearly empty start moving toward the glass who communicated the event. If the glass is taken back or manipulated by an user, the action is aborted. If not, the glass continues its displacement avoiding obstacles. When sufficiently close to the original glass, they stop, show by a LED that they are ready to be filled and wait until they are refilled. Then they move back to their original position. If the glass is placed too close to the table border, the glass takes the initiative to go back to its original position.

An alternative scenario involves a carafe robject. In this case the detection of the filling action is done when the carafe is taken and not when the glass starts to be filled. 


\subsection{Current state of the art}

Very few research groups have started working on distributed robotic systems acting in the living environment, what they call ubiquitous robotics. The most active group is leaded by Prof. Kim and started to work on this topic in 2004 [2, 3, 4, 5]. Their vision of ubiquitous robotics is based on the communication between intelligent software agents (Sobots), sensors networks (Embots) and classical mobile robot (Mobots). Sobots represent the intelligence, Embots the sensor part of the system and Mobots the actuator part. User interaction is not playing a central role in their concept. The combination of these domains is apparently only a way of generating a new application field for the three technologies, without a real integration.

Another attempt at defining ubiquitous robotics has been made by Takeda [6] with a stronger emphasis on interaction. This paper introduces a very interesting vision including specific interaction modes such as "intimate interaction", "loose interaction" and "cooperative interaction". This work has been limited to some experiments, without a real integration effort and a development of concepts and technology.

Nobody has developed mobile drinking glasses, but some systems look for similar services, like the BrewskiBot of MobileRobots Inc. It's a mobile fridge which

brings some drink to people. In fact it's a little fridge fixed on a PatrolBot, a robot design to patrol in an area.

\section{The mechatronic system}

\subsection{Specifications}

To carry out the selected scenario, our robjects must respect the following specifications.

- To contain $3 \mathrm{dl}$ of liquid. Its form has to be adapted to the content.

- To move without spill the content.

- To draw the attention with a luminous or sonorous signal.

- To detect an impact with an acceleration higher of $1 \mathrm{~g}\left(10 \mathrm{~m} / \mathrm{s}^{2}\right)$ and its direction with a precision of more or less $10^{\circ}$.

- To detect an obstacle placed within 2 centimeter around the robot.

- To orient itself according to the carafe and the user with a precision of $2 \mathrm{~cm}$ and about $5^{\circ}$.

- To detect liquid level in the glass (about ten different levels for a glass of 30cl).

- To detect when someone lift up the carafe.

- To detect the border of the table in order to avoid falling from the table.

- To detect when the glass is too close (less than $15 \mathrm{~cm}$ ) to the table border. 


\subsection{Selected solution}

After an analysis of several options and constrains of the project (duration, competences involved) we decided to stick a common drinking glass on the top of a flat mobile robot. The robot has two differential wheels to move on the table. We use LEDs to draw the attention of users. The robot has an accelerometer sensor to detect impacts. Around his body, height infrared proximity sensor are available to detect obstacles. We use a tracking system based on the ArTag Library [7] and a high definition camera connected with a computer to detect the positions of the robots. The computer can exchange information with the robots using Bluetooth devices. We use a force sensor as a balance to check the liquid level. The carafe is also placed on a robotic element to detect when someone lift it up. This robotic element of the carafe has a force sensor and can exchange information with Bluetooth but cannot move. The table border is detected by the high-definition camera which is already used to find the position of glasses.

\subsection{Structure of the system}

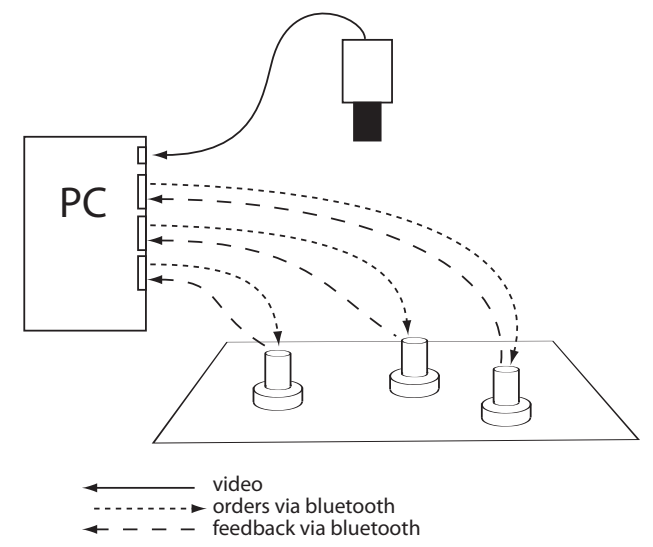

Fig. 2 Structure of the system with a PC controlling the robjects and observing their position using a camera.

Despite the long-term purpose to design a fully distributed system, this first attempt has been based on a centralized approach, at least at the structural level. A PC has the control of the whole setup, taking images from the camera, extracting the position of the glasses in respect to the table shape and controlling the glasses based 
on the feedback they give (see figure 2). The glasses robjects are still in charge of all sensor pre-processing, detection of events and motor control.

\subsection{Mechanics of the robjects}
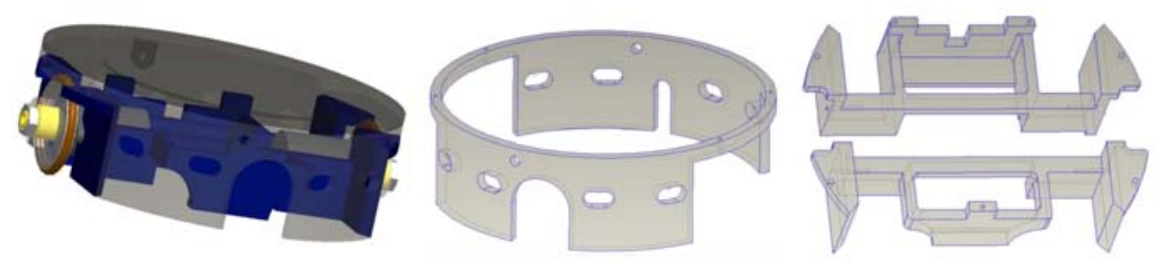

Fig. 3 Proe drawings of the mecanical part

The robject has the following configuration: It is a glass fixed on a small cylindrical mobile robot having $10 \mathrm{~cm}$ of diameter and $3.4 \mathrm{~cm}$ high (figure 3 ). The robot has two differential wheels with a diameter of $2.6 \mathrm{~cm}$. The part of the robot that is glued to the glass can move around an axis and acts on a force sensor as a balance. The same mechanical part protects the electronic of spatter. Figure 4 shows the mechanism of the balance. A plexiglas frame (blue part in figure 3) fixes the battery, motors and the print circuit board.

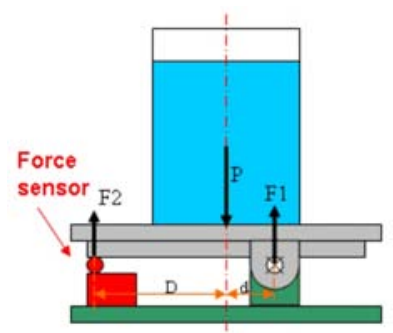

Fig. 4 Geometrical configuration of the balance.

Wheel are actuated by stepper motors with 20 steps by revolution and a 50:1 gearbox. The torque is sufficient to move the robot with a payload of one kilogram. 


\subsection{Electronics of the robjects}

The electronics of the robot is based on the electronics of the e-puck robot ${ }^{1}$.

The microcontroller used for controlling the robot is a DSPIC30F by Microchip running at 15 MIPS. It has $68 \mathrm{I} / \mathrm{O}$ pins of which 12 can perform analogical/digital conversion and it support two UART communications channels.

To detect obstacles, we use infrared proximity sensors. Height proximity sensors are placed symmetrically to the forward axis with angles of $20^{\circ}, 45^{\circ}, 135^{\circ}$ and $160^{\circ}$. It could have been useful to place a sensor at $90^{\circ}$ but unfortunatly wheels prevented from placing the sensor at this position. The frame's plexiglas sidetracks a part of the infrared sensor's ray which is reflected on the floor. This can cause troubles if the distance between the plexiglas and the floor changes, because the sensor's value changes too. We exploited this aspect to detect when the plexiglas has passed the table's border.
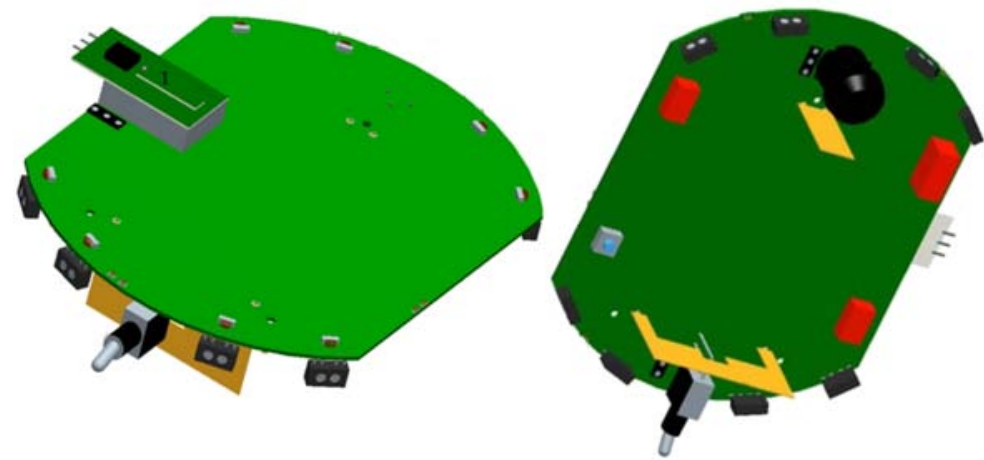

Fig. 5 CAD drawings of the pcb's top and pcb's bottom

To measure the liquid level and to know when the glass is lift up we use the "millinewton" force sensor [8]. This sensor returns a voltage functions of the force applied on a contact ball. The sensor saturates with a force of about $2 \mathrm{~N}$. To know the position in space of the robot and to detect impacts we use a 3D accelerometer sensor. With this sensor and the force sensor we can know if the glass is lifted by the user. To communicate with the user, the robot has eight horizontal red LEDs on the top and four vertical yellow LEDs. We can command each red LEDs individually. All yellow LEDs are controlled together. To exchange information with the computer or receive order from the computer, the robot has a Bluetooth device. The robot has a lithium-ion battery for supplying with a voltage of $3.7 \mathrm{~V}$ and a capacity of $1400 \mathrm{mAh}$. With this battery the robot has an autonomy of about two hours when the motors are continously active.

\footnotetext{
${ }^{1}$ http://www.e-puck.org
} 


\section{Software}

The main software runs on the PC computer and receives information from the robjects and from the high-definition camera fixed over the table. From camera images we extract the location of all glasses and of the table border. Using bluetooth, the PC sends orders to the robots as function of the received information obtained by the camera and from the robots. The software running on the robots receives and executes order received from the PC. Some basic algorithms to do obstacle avoidance, table's border detection, pre-processing of force sensor data (if someone takes the glass, if the glass is empty, if someone fill up or drain the glass, if the liquid level laze) are embedded in the robots themselves.

\subsection{Image processing and selected camera}

To know the location and the direction of each glass and of the carafe, we apply visual tracking using the ArTag library [7]. This library was mainly developed for augmented virtual reality and works with 2D tags which are found in the image and identified. We can create objects with many tags having a fixed geometrical relation among them. The software must recognize at least two tags to identify an object and its location.

The robots have twelve tags on the tray around the glass; with this configuration we expect that the camera can see at least two tags which are not hidden by the glass itself or by the user. Unfortunately at this location there is not a lot of space and tags are small $(15 \times 15 \mathrm{~mm})$. This size defines the required resolution of the camera: if we want to analyze a square table with a size of $1.5 \times 1.5 \mathrm{~m}$, we must use a camera with at least a resolution of 2000x2000 pixels. To minimize radial distortion we use a lens with a focal of $12 \mathrm{~mm}$ and we choosed an integration time of 250 milliseconds to increase picture quality. With this configuration, the delay between image acquisition and position computation is about one second. Therefore odometry is combined with the tracking system to get real-time position values.

\subsection{Embedded system}

The software running on the robots allows them to autonomously (i) move forward for a given distance avoiding obstacles and table's borders, (ii) turn for a given angle, (iii) switch on or off LEDs, (iv) calibrate proximity sensors, (v) calibrate the balance and sent the balance's state (empty, carried, filling up, emptying, stagnant) or the balance's value, and (vi) send the motion detector's value. 


\subsection{Main control}

The main control runs on the computer, manages all the system and send orders to the robots by Bluetooth. When launched, the software starts by performing an initialization of the camera and of the ArTag library, a connection with the glass robots and the carafe robots, detection of the table border and of the robots location.

Then the software runs in a loop and controls each glass as following:

- If the glass is at its location and is empty, the software check if someone fills up another glass (A). In this case the software sends orders to move the glass toward the glass (A).

- If the glass is not moving and somebody moves it, the software controls the new location. If the glass is too close to the table border, the software sends the order to move the glass toward is last location, otherwise it record the new location.

- If the glass is moving to be filled up and somebody takes it, the software checks the new position. If the glass is nearer to its standard location than to the place where it must be filled up, the software send the order to move back to its standard location. Otherwise the glass will continue its displacement.

- When the glass reaches the place where it must be filled up, it waits for somebody filling it up and then returns toward is location.
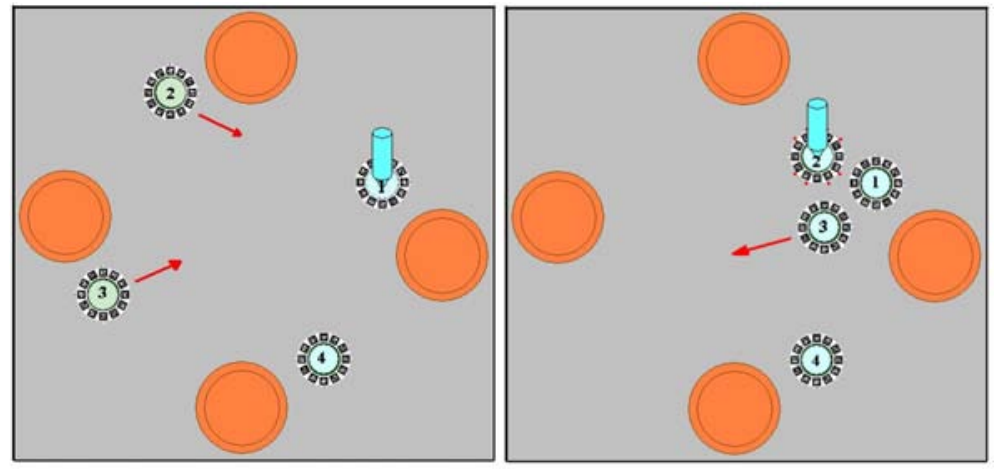

Fig. 6 On the left: the glass 1 is filling up and the two empty glasses move to it, On the right: the glass 3 was filling up and returns to its location.

\section{Validation in real situations}

Three experiments were done on a square table with a size of $1.2 \times 1.2$ meters. The first experiment was done with three persons who had each a glass of water. The second experiment has been carried out with four persons with a glass of water 


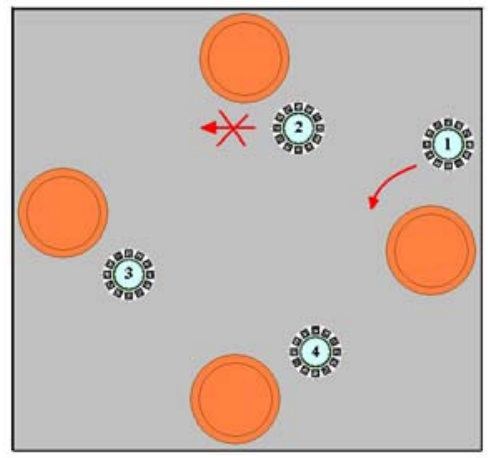

Fig. 7 Somebody moved glasses 1 and 2. Glass 1 is too close to the table border and returns to its last location, glass 2 don't move and record its new location

each. The third experiment looked to four persons as in the original scenario, two having only a glass of water, two having both water and wine glasses. Users had no idea about how the system works and got only few explanations (one minute) before the start of the experiment.

\subsection{Users' feeling}

Users have given their feeling about the system, mainly on usefulness, readiness and speed of the system.

- It is easy to interact with the glass. The action to catch the glass when it moves toward the filling place if the user don't wont to drink anymore is really intuitive. It's nice to see the glasses coming when somebody start filling. It's natural.

- The system is not fast enough. A speed of $8 \mathrm{~cm} / \mathrm{s}$ is not sufficient. The speed of mobile robots when interacting with humans is a common problem in interaction with robotics infrastructures [9]. In our case, when somebody finished to fill up his glass, the other glasses were still at the half of their trajectory. This delay destroys the efficiency of the interaction.

- Wrong robot's actions (avoiding non-existent obstacles, ...) distract the users who don't know how to react.

- Since the glass is glued on the robot, users have to lift all the system, which looks strange because of the impression that the robot could fall. Users get quickly used to this wrong impression.

- This system don't allow to follow the civility's rule which says that we must serve other persons before serving ourself, or, like in Japan, that we cannot serve ourself! 


\section{Conclusion}

The positive conclusion of our experiments is that the robject concepts seems very interesting and allowing natural interactions between robots and users in everyday life. The negative aspect is that this type of interaction must be precise, reliable and fast. This is still not the case of our system. The main interaction problem is clearly generated by wrong robots' actions: sometimes robots misinterpret signals and react in the wrong way, generating confusion in the user. That's due mainly to the simplicity of the embedded sensors. Another big problem is the speed of the system, not sufficient to implement an optimal interaction. On the other side, this project demonstrates that we can use simple, intuitive and usual actions to control robots. For example filling up glasses, catching a moving glass, placing it on standard positions etc. This interaction allows everybody to accept and use robotic technology embedded in everyday objects.

Acknowledgements We would like to thanks Luc Bergeron and Frédéric Kaplan for their suggestions, ideas and support, Tarek Baaboura for parts manufacturing, ACI staff particulary Peter Brühlmeier for pcb routing and André Badertscher for components soldering, Michael Bonani for advice in electronic, and all testers for their patience and very valuable feedback.

\section{References}

1. Céline Ray, F. Mondada, and R. Siegwart. What do people expect from robots? In IEEE/RSJ 2008 International Conference on Intelligent RObots and Systems.

2. Jong-Hwan Kim. Ubiquitous robot. In Computational Intelligence, Theory and Applications, Keynote speech paper of the 8th Fuzzy Days International Conference, pages 451-459. Springer, 2004.

3. Jong-Hwan Kim, Yong-Duk Kim, and Kang-Hee Lee. The 3rd generation of robotics: Ubiquitous robot. In Proc. of the International Conference on Autonomous Robots and Agents (Keynote Speech Paper), 2004.

4. Jong-Hwan Kim. Ubiquitous robot: Recent progress and development. In ICASE International Joint Conference 2006 (Keynote Speech Paper), pages I-25 - I-30, 2006.

5. Jong-Hwan Kim, Kang-Hee Lee, Yong-Duk Kim, N.S. Kuppuswamy, and Jun Jo. Ubiquitous robot: A new paradigm for integrated services. In Robotics and Automation, 2007 IEEE International Conference on, pages 2853-2858, 2007.

6. H. Takeda, N. Kobayashi, Y. Matsubara, and T. Nishida. Towards ubiquitous human-robot interaction. In Working Notes for IJCAI-97 Workshop on Intelligent Multimodal Systems, pages $1-8,1997$.

7. Mark Fiala. Artag, a fiducial marker system using digital techniques. In $C V P R$ (2), pages 590-596. IEEE Computer Society, 2005.

8. H Birol, T Maeder, I Nadzeyka, M Boers, and P Ryser. Fabrication of a Millinewton Force Sensor Using Low Temperature Co-fired Ceramic (LTCC) Technology. Sensors and Actuators A: Physical, 134:334-338, 2007.

9. F. Mondada and S. Legon. Interactions between Art and Mobile Robotic System Engineering. In Proceedings of the International Symposium on Evolutionary Robotics From Intelligent Robotics to Artificial Life, Lecture Notes In Computer Science, pages 121 - 138. Springer, 2001. 